VOL. 60 (1999) [349-350]

\title{
Theorems of Birkhoff type in pseudovarieties and e-varieties of regular semigroups
}

\section{Genevieve Churchill}

This thesis is concerned with the problem of being able to use, or generalize, Birkhoff's fundamental theorems for classes of algebras which do not form varieties - particularly in pseudovarieties and e-varieties. We first look at pseudovarieties and certain generalized varieties.

Let $\mathcal{C} o m, \mathcal{N}$ il and $\mathcal{N}$ denote the generalized varieties of all commutative, nil, and nilpotent semigroups respectively. For a class $\mathcal{W}$ of semigroups let $\mathcal{L}(\mathcal{W})$ and $\mathcal{G}(\mathcal{W})$ denote respectively the lattices of all varieties and generalized varieties of semigroups contained in $\mathcal{W}$. In [1] Almeida, having shown that the mapping $\mathcal{L}(\mathcal{N}$ il $\cap \mathcal{C}$ om $) \cup$ $\{\mathcal{N} i l \cap \mathcal{C o m}\} \rightarrow \mathcal{G}(\mathcal{N} \cap \mathcal{C} o m)$ given by $\mathcal{W} \mapsto \mathcal{W} \cap \mathcal{N}$ is an isomorphism, asked whether the extension of this mapping to $\mathcal{L}(\mathcal{N} i l) \cup\{\mathcal{N} i l\}$ is also an isomorphism.

In the first part of the thesis this question is considered. We initially show that the extension is not surjective. Non-injectivity is then established; this involves analysing sequences of words of unbounded lengths derived from the defining identities of certain nil varieties. Results of a more general nature are also given, involving the question of when two arbitrary semigroup varieties possess the same set of nilpotent semigroups.

In the second part of the thesis we turn to the problem of establishing analogues of Birkhoff's theorem for e-varieties. Auinger's Birkhoff-style theory for locally inverse e-varieties, developed in [2] and [3], is expanded in order to obtain a unified theory for e-varieties of locally inverse or of $E$-solid semigroups - by Yeh's result from [8] this is the entire lattice of e-varieties in which nonmonogenic bifree objects exist. In addition an alternative unification is given, based on the techniques used by Kadourek and Szendrei in [7] to describe a Birkhoff-style theory for $E$-solid e-varieties.

We proceed to show that trifree objects on at least three generators exist in an e-variety $\mathbf{V}$ of regular semigroups if and only if $\mathbf{V}$ is locally $E$-solid; this extends Kadourek's work in [6] on the existence of trifree objects in locally orthodox e-varieties and generalizes Yeh's result on the existence of bifree objects.

Received 21st June, 1999

Thesis submitted to the University of Tasmania, October 1998. Degree approved, December 1998. Supervisor: Dr. P. Trotter.

Copyright Clearance Centre, Inc. Serial-fee code: 0004-9727/99 \$A2.00+0.00. 
In conclusion, a theory of " $n$-free" objects is outlined, indicating how analogues of the concept of a free object can be defined for any e-variety.

The results given in the first part of the thesis have appeared in [4]. The results of the second part are to appear in [5].

\section{REFERENCES}

[1] J. Almeida, Finite semigroups and universal algebra (World Scientific, Singapore, 1995).

[2] K. Auinger, 'The bifree locally inverse semigroup on a set', J. Algebra 166 (1994), 630-650.

[3] K. Auinger, 'On the bifree locally inverse semigroup', J. Algebra 178 (1995), 581-613.

[4] G. Churchill, 'Almeida's generalized variety problem', J. Algebra 196 (1997), 499-519.

[5] G. Churchill and P.G. Trotter, 'A unified approach to biidentities for e-varieties', Semigroup Forum (to appear).

[6] J. Kadourek, 'On some existence varieties of locally orthodox semigroups', Internat. J. Algebra Comput. 7 (1997), 93-131.

[7] J. Kadourek and M.B. Szendrei, 'On existence varieties of $E$-solid semigroups', Semigroup Forum 58 (1999), 17-68.

[8] Y.T. Yeh, 'The existence of bifree objects in e-varieties of regular semigroups', Internat. J. Algebra Comput. 2 (1992), 471-484.

Department of Mathematics

University of Tasmania

Hobart Tas 7000

Australia 